\title{
Progresos en el control de la infección por el VIH y el sida en Navarra, 1985-2003
}

\section{Progress in the control of HIV infection and AIDS in Navarre, 1985-2003}

\author{
C. Moreno-Iribas' ${ }^{1}$, F. Irisarri' ${ }^{1}$, L. Elizalde ${ }^{1}$, M. Urtiaga ${ }^{1}$, J. Sola ${ }^{2}$, C. Fernández Jauregui ${ }^{3}$, V. \\ Martínez De Artola $^{4}$, J. Sáinz de Murieta ${ }^{5}$, I. Dorronsoro ${ }^{3}$, T. Rubio ${ }^{5}$, J. Castilla ${ }^{1}$
}

\section{RESUMEN}

Se revisa la situación epidemiológica de la infección por el VIH y el sida en Navarra en 2003

Hasta diciembre de 2003 se habían diagnosticado 1.610 infecciones por el VIH en residentes en Navarra, de los cuales el $41 \%$ habían fallecido. Los nuevos diagnósticos de $\mathrm{VIH}$ han disminuido un $81 \%$ entre 1993 y 2003 , año en que se diagnosticaron 28 casos (4,8 por 100.000 habitantes). El descenso se produjo fundamentalmente en las infecciones en usuarios de drogas inyectadas, ya que los casos por transmisión sexual se han mantenido estables. Más de la mitad de las infecciones diagnosticadas en el período 2000-2003 (58\%) eran atribuibles a transmisión heterosexual, el $18 \%$ ocurrieron en usuarios de drogas por vía parenteral y el $12 \%$ en hombres homosexuales. El 33\% eran personas originarias de otros países. La incidencia de sida ha disminuido de 75 casos en 1996 a 20 en 2003, y la mortalidad de 65 a 8 casos, respectivamente. En el período 2000-2003 la incidencia media anual de sida fue de 4,2 por 100.000 habitantes y la tasa media anual de mortalidad de 1,6 por 100.000 habitantes.

A finales de 2003 había 902 personas vivas con diagnóstico de VIH seguidas en el sistema sanitario (1,6 infecciones conocidas por 1.000 habitantes).

En 2003 el 65\% de los jóvenes de 15 a 29 años refería relaciones sexuales coitales, porcentaje mayor que en años anteriores, pero su nivel de información sobre prevención del sida fue aceptable. Es preciso insistir en la prevención y adaptarla a las nuevas situaciones.

Palabras clave. Sida. VIH. Vigilancia epidemiológica. Incidencia.

An. Sist. Sanit. Navar. 2004; 27 (2): 221-231.

1. Instituto de Salud Pública de Navarra

2. Servicio de Enfermedades Infecciosas. Hospital de Navarra

3. Servicio de Microbiología. Hospital de Navarra

4. Sección de Microbiología. Hospital Virgen del Camino

5. Servicio de Medicina Interna. Hospital García Orcoyen

Aceptado para su publicación el 10 de junio de 2004.

\section{ABSTRACT}

The epidemiological situation of HIV infection and AIDS in Navarre in 2003 is reviewed.

Up until December 2003, 1,610 HIV infections had been diagnosed in residents of Navarre, of whom $41 \%$ had died. The new diagnoses of HIV fell by some $81 \%$ between 1993 and 2003, a year in which 28 cases were diagnosed (4.8 per 100,000 inhabitants). The fall basically occurred in infections in injection drug users, since the cases due to sexual transmission had remained stable. Over half of the infections diagnosed in the period 2000-2003 (58\%) were attributable to heterosexual transmission, $18 \%$ occurred in parenteral drug users and $12 \%$ in homosexual men. Thirty-three percent were persons originally from other countries. The incidence of AIDS fell from 75 cases in 1996 to 20 in 2003 , and mortality from 65 to 8 cases, respectively. In the 2000-2003 period, the average annual incidence of AIDS was 4.2 per 100,000 inhabitants and the average annual rate of mortality was 1.6 per 100,000 inhabitants.

At the end of 2003, there were 902 living persons with a diagnosis of HIV monitored by the health system (1.6 known infections per 1,000 inhabitants).

In $2003,65 \%$ of the youths aged between 15 and 29 referred to coital sexual relations, a higher percentage than in previous years, but their level of information on the prevention of AIDS was acceptable. It is necessary to insist on prevention and to adapt this to the new situation.

Key words. AIDS. HIV. Epidemiological surveillance. Incidence.

\section{Correspondencia:}

Conchi Moreno Iribas

Instituto de Salud Pública

C/ Leyre 15

31003. Pamplona

Tfno: 848423470

email:mmorenoi@cfnavarra.es 


\section{INTRODUCCIÓN}

Han transcurrido dos décadas desde la detección de los primeros casos de sida en Navarra y, durante este tiempo, se ha producido un elevado número de infecciones y de fallecimientos por esta causa ${ }^{1,2}$. Desde los primeros momentos se emprendieron numerosas actividades tratando de evitar la transmisión de la infección, mejorar el estado de salud de las personas infectadas y conseguir su normalización en la sociedad. El curso de esta epidemia está influenciado por múltiples circunstancias y factores de tipo biológico, demográfico, social, conductual, económico y médico. Sobre ella han incidido intervenciones del sistema de salud, pero también de otras administraciones no sanitarias, como las educativas, de las organizaciones no gubernamentales y de diferentes grupos sociales ${ }^{3}$. Las intervenciones con las que podemos modificar el curso de la epidemia de VIH pueden agruparse en tres tipos:

1. La prevención de la transmisión del $\mathrm{VIH}$, que requiere el mantenimiento de conductas seguras en la población a lo largo del tiempo.

2. El diagnóstico de la infección, el cual posibilita la prevención de forma dirigida y el acceso a cuidados.

3. La atención sanitaria, psicológica y social a las personas infectadas, con el objeto de mejorar su supervivencia y calidad de vida.

La epidemia de VIH y sida está configurada en la población por un amplio espectro de situaciones, que abarcan desde las personas que se exponen a riesgo, pasando por las personas asintomáticas que viven con la infección por el VIH, hasta aquellas que han llegado a presentar patologías graves asociadas a la inmunodeficiencia.

La información epidemiológica ha de informar sobre el curso de la epidemia y sobre las características de las personas afectadas, con el objetivo último de servir para orientar las actividades de lucha contra el sida. Para ello se requiere que se monitoricen al menos los siguientes aspectos:
1. Los comportamientos de riesgo para la infección por el VIH y sus factores condicionantes, sobre los cuales se pueda incidir a través de estrategias de promoción de la salud.

2. El número y descripción de las personas que adquieren la infección por el VIH. Esta información es necesaria para detectar las necesidades en materia de prevención. Ante la dificultad para conocer el momento en que se ha producido la infección, se suele realizar una aproximación a través del análisis de las infecciones por VIH diagnosticadas.

3. La dimensión y características de la población que vive infectada por el VIH. Tiene interés su caracterización epidemiológica y su situación clínica. Su estudio se ve habitualmente limitado a aquellas personas cuya infección ha sido diagnosticada.

4. Las consecuencias graves para la salud, incluyendo la morbilidad y mortalidad.

A la vista de todo lo anterior es evidente que se requiere distintos tipos de información para describir todos los aspectos de interés de esta epidemia. Ello obliga a que la vigilancia epidemiológica deba basarse en diferentes abordajes complementarios, que al menos cubran el estudio de las conductas de riesgo, la vigilancia de la infección por el VIH, la de casos de sida y el estudio de la mortalidad ${ }^{1}$.

En el presente trabajo se revisa la situación epidemiológica y los indicadores disponibles en Navarra hasta finales de 2003.

\section{MATERIAL Y MÉTODOS}

Las fuentes de información para la realización de este estudio han sido los sistemas de vigilancia epidemiológica de la infección por el VIH y el sida en Navarra². Ambos sistemas son coordinados por el Instituto de Salud Pública y abarcan toda la población navarra a lo largo de todo el período desde el comienzo de la epidemia. Además se incorporan los resultados sobre conductas de riesgo obtenidos en dos encuestas realizadas a jóvenes y a usuarios de drogas por vía parenteral ${ }^{4,5}$. 
La vigilancia epidemiológica de la infección por VIH se realiza en Navarra mediante un sistema de notificación de diagnósticos de esta infección, que sigue los criterios de definición de caso y la metodología recomendada por el Centro Europeo para la Vigilancia Epidemiológica del VIH y sida y por los Centros de Control de las Enfermedades de los EE UU ${ }^{6-9}$. No obstante, este sistema no ha logrado implementarse en algunos países de Europa ${ }^{7}$, y dentro de España, hasta el momento, sólo se realiza en algunas comunidades autónomas ${ }^{1,8}$.

Se considera diagnóstico de infección por el VIH la presencia de anticuerpos específicos confirmados mediante una prueba de alta especificidad, habitualmente Western blot, independientemente de la presencia o ausencia de síntomas o del estadio clínico. En niños recién nacidos ha de demostrarse la persistencia de los anticuerpos pasados 18 meses o bien demostrarse la presencia del virus. ${ }^{6,8}$ El diagnóstico de la infección por el VIH es un diagnóstico de laboratorio, pudiendo darse el caso de personas que se repiten el diagnóstico en más de una ocasión y el de otras personas infectadas que permanecen sin diagnosticarse ${ }^{10}$.

Las notificaciones de VIH en Navarra proceden fundamentalmente de los laboratorios que realizan la prueba de confirmación y de los servicios hospitalarios que atienden a pacientes con infección por el VIH. En el caso de personas con más de un diagnóstico de VIH se consideró la fecha del primero. Esta notificación se completa con la búsqueda activa de casos. Además se realiza seguimiento activo de los pacientes incluidos, con el objeto de verificar el diagnóstico de $\mathrm{VIH}$, establecer si reside habitual o temporalmente en Navarra, si se ha producido el diagnóstico de sida, el estado vital, y el lugar donde tiene lugar el seguimiento clínico. Para este seguimiento activo se consultan el registro de casos de sida, el registro de tuberculosis, el registro de mortalidad, las determinaciones realizadas en el Servicio de Microbiología del Hospital de Navarra y del Hospital Virgen del Camino, los registros de urgencias, el conjunto mínimo básico de datos al alta hospitalaria y la base de datos de la tarjeta sanitaria y el padrón.
Para completar y corroborar la información se han revisado las historias clínicas de los pacientes ${ }^{2}$.

La vigilancia epidemiológica de los casos de sida se realiza en toda Europa siguiendo una metodología y definición de caso similares. Se considera caso de sida a toda persona diagnosticada de infección por el VIH que presenta o ha presentado alguna "enfermedad definitoria de sida" Estas enfermedades incluyen infecciones oportunistas, algunos cánceres, la demencia y el síndrome caquéctico por VIH. La clasificación como caso de sida es definitiva, independientemente de la reversión del cuadro clínico que ocasionó el diagnóstico. Los casos son declarados por los médicos que realizan el diagnóstico, y el sistema se completa mediante búsqueda activa de casos utilizando las mismas fuentes que para el VIH.

Los sistemas de vigilancia epidemiológica del VIH y de sida se crearon al amparo de la legislación sanitaria del Estado y de Navarra, y por contener datos personales e individualizados se someten a la Ley Orgánica de Protección de Datos de 1999 (LOPD).

Los análisis se han centrado en los casos que tenían residencia habitual en Navarra. El período de estudio ha abarcado desde 1985 hasta diciembre de 2003. Se ha comparado la tasa de diagnósticos anuales de VIH en Navarra con la de otros países de Europa que cuentan con sistemas de información con características similares.

Para evaluar la frecuencia de conductas de riesgo se han utilizado datos de dos encuestas realizadas por la Fundación Bartolomé de Carranza. Una de ellas consiste en cuatro cortes transversales en los años, $1982,1991,1998$ y 2003, sobre la conducta sexual de los jóvenes navarros de 15 a 21 años $^{4}$. La segunda, fue realizada en 2002 y se dirigió a explorar los conocimientos y conductas de consumidores activos de drogas ${ }^{5}$.

\section{RESULTADOS}

\section{Personas diagnosticadas de infección por el VIH entre 1985 y 2003}

Hasta diciembre de 2003 se habían diagnosticado un total de 2.385 personas 
con infección por el VIH en Navarra, después de haber excluido diagnósticos repetidos y pruebas no confirmadas. En 1.610 casos los datos censales y de la historia clínica indicaban que el paciente tenía residencia habitual en Navarra, mientras que $775(32,5 \%)$ eran personas que residían temporalmente (Fig. 1). En este último grupo se incluyeron principalmente personas que han residido en Navarra uno o varios años para recibir tratamientos de deshabituación de drogas en centros como Remar o el Patriarca.

A finales de 2003 habían fallecido 665 $(41,3 \%)$ de las 1.610 personas diagnosticadas de infección por VIH que residían en Navarra. En otros 43 casos no pudo confirmarse ni descartarse el fallecimiento. De los 902 que permanecían vivos, su relación con los servicios asistenciales era la siguiente: una gran mayoría (73\%) estaba siendo atendido en el Servicio de Infecciosas del Hospital de Navarra y el resto estaba siendo seguido en los Servicios de Medicina Interna de los Hospitales García Orcoyen, Reina Sofía y Virgen del Camino, en atención primaria u en otros dispositivos asistenciales. Además había 36 casos que se habían trasladado fuera de Navarra (Fig. 1).

\section{Tendencia de nuevos diagnósticos de VIH}

Los nuevos diagnósticos de infección por el VIH alcanzaron las tasas máximas entre 1987 y 1993, con más de 20 nuevos diagnósticos anuales por cada 100.000 habitantes durante todo este período. Desde entonces el número de diagnósticos de VIH anuales ha disminuido un $81 \%$, hasta situarse en 4,8 casos por 100.000 habitantes en 2003. La tendencia se mantiene descendente con una disminución media del 7,5\% anual durante los últimos cinco años (Tabla 1).

Este descenso se debe fundamentalmente a la importante caída en los diagnósticos de infecciones por $\mathrm{VIH}$ adquiridas por compartir material para el consumo de drogas inyectadas, que ha dejado de ser la principal vía de transmisión del VIH desde 1996 (Fig. 2). Un cambio similar se ha descrito en otros lugares de España ${ }^{1,12,13}$. Tanto los casos de infecciones atribuibles a transmisión heterosexual como los de transmisión por prácticas homosexuales entre hombres se mantienen en niveles relativamente estables. Desde 1998 no se ha diagnosticado ninguna infección por el VIH en niños.

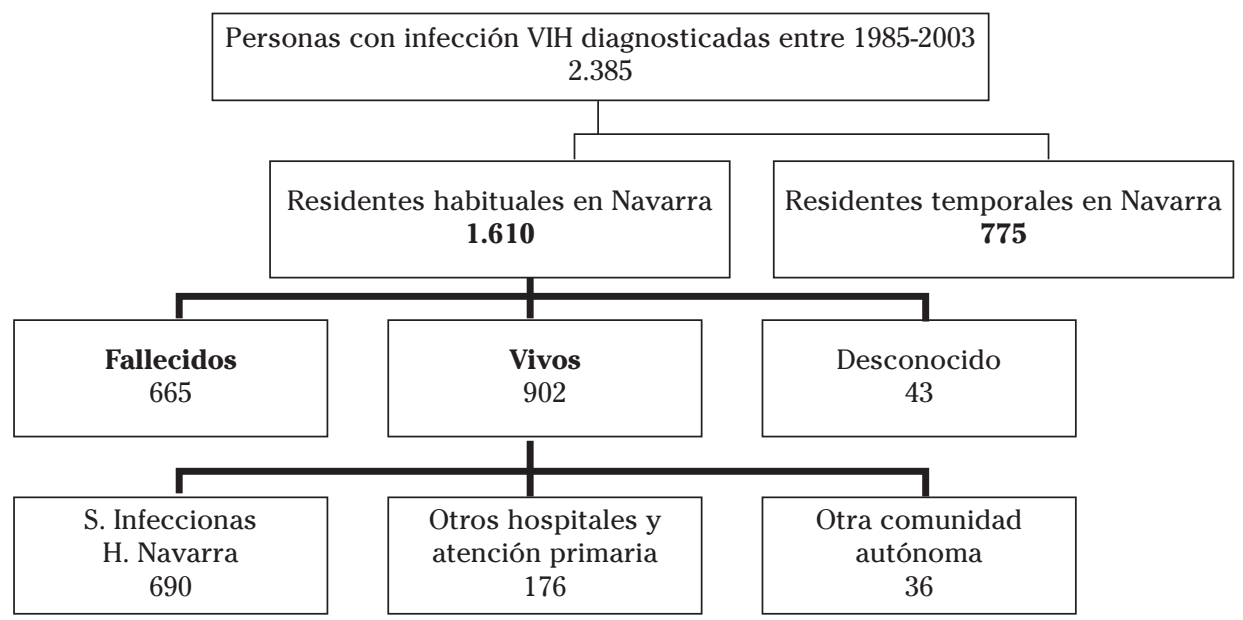

Figura 1. Situación a diciembre de 2003 de las personas diagnosticadas de infección por el VIH en Navarra. 
Tabla 1. Evolución anual del número y de las tasas por millón de habitantes de nuevos diagnósticos de VIH, de casos de sida y de fallecimientos por sida en personas residentes en Navarra, 1985-2003.

\begin{tabular}{|c|c|c|c|c|c|c|}
\hline \multirow[t]{2}{*}{ Año } & \multicolumn{2}{|c|}{ Diagnósticos de VIH* } & \multicolumn{2}{|c|}{ Diagnósticos de sida** } & \multicolumn{2}{|c|}{ Muertes por sida** } \\
\hline & Casos & Tasas por millón & Casos & Tasas por millón & Casos & Tasas por millón \\
\hline 1985 & 103 & 198 & 2 & 4 & 1 & 2 \\
\hline 1986 & 103 & 198 & 4 & 8 & 1 & 2 \\
\hline 1987 & 165 & 318 & 12 & 23 & 8 & 15 \\
\hline 1988 & 104 & 200 & 24 & 46 & 8 & 15 \\
\hline 1989 & 115 & 221 & 20 & 39 & 19 & 37 \\
\hline 1990 & 120 & 231 & 44 & 85 & 24 & 46 \\
\hline 1991 & 144 & 276 & 50 & 96 & 21 & 40 \\
\hline 1992 & 131 & 251 & 62 & 119 & 35 & 67 \\
\hline 1993 & 108 & 206 & 81 & 155 & 39 & 74 \\
\hline 1994 & 84 & 160 & 90 & 171 & 64 & 122 \\
\hline 1995 & 84 & 159 & 89 & 169 & 62 & 117 \\
\hline 1996 & 58 & 110 & 75 & 142 & 65 & 123 \\
\hline 1997 & 51 & 96 & 44 & 83 & 29 & 55 \\
\hline 1998 & 58 & 109 & 43 & 81 & 15 & 28 \\
\hline 1999 & 40 & 74 & 36 & 67 & 14 & 26 \\
\hline 2000 & 38 & 70 & 24 & 44 & 11 & 20 \\
\hline 2001 & 41 & 74 & 19 & 34 & 10 & 18 \\
\hline 2002 & 34 & 60 & 31 & 54 & 9 & 16 \\
\hline 2003 & 28 & 48 & 20 & 35 & 8 & 14 \\
\hline
\end{tabular}

${ }^{*}$ Fuente: registro de $\mathrm{VIH}$.

**Fuente: registro de sida.

A lo largo de toda la epidemia los diagnósticos de VIH en hombres han superado en 2,4 veces a los de mujeres. En el período 2000-2003 el número de nuevos diagnósticos de VIH ha seguido descendiendo en hombres, pero se ha estancado en mujeres, de forma que la razón hombre/mujer ha pasado a ser de 1,8.

En los últimos años, y coincidiendo con los cambios demográficos, la proporción de casos de VIH en personas originarias de otros países está aumentando (Fig. 3), siguiendo una tendencia similar a la de otros países de Europa occidental ${ }^{14,15}$.

La tasa de nuevos diagnósticos de VIH en Navarra se situaba a mediados de los noventa en un rango alto en comparación con la de otros países de Europa. Sin embargo, en Navarra han disminuido los nuevos diagnósticos de VIH más que en otros países, ya que incluso en algunos como Bélgica, Irlanda y Reino Unido, estos

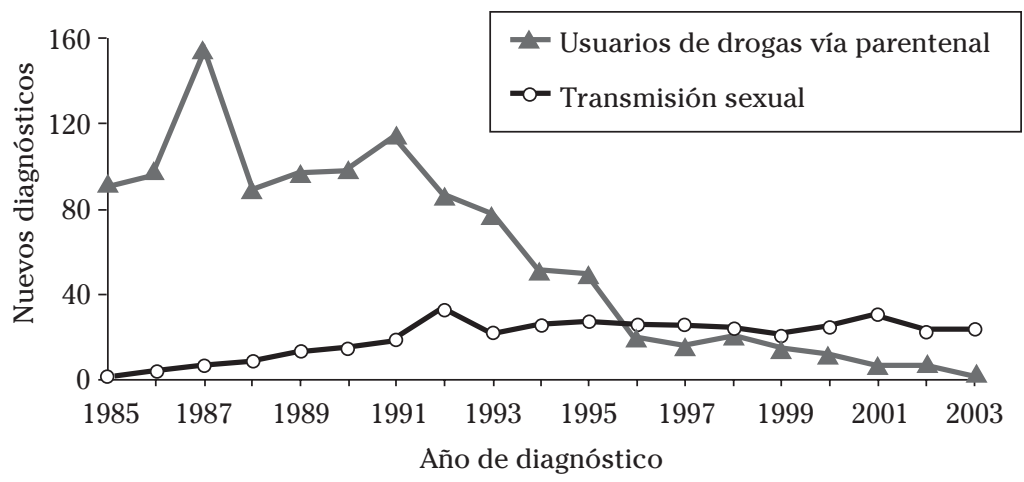

Figura 2. Nuevos diagnósticos de VIH según categoría de transmisión. 


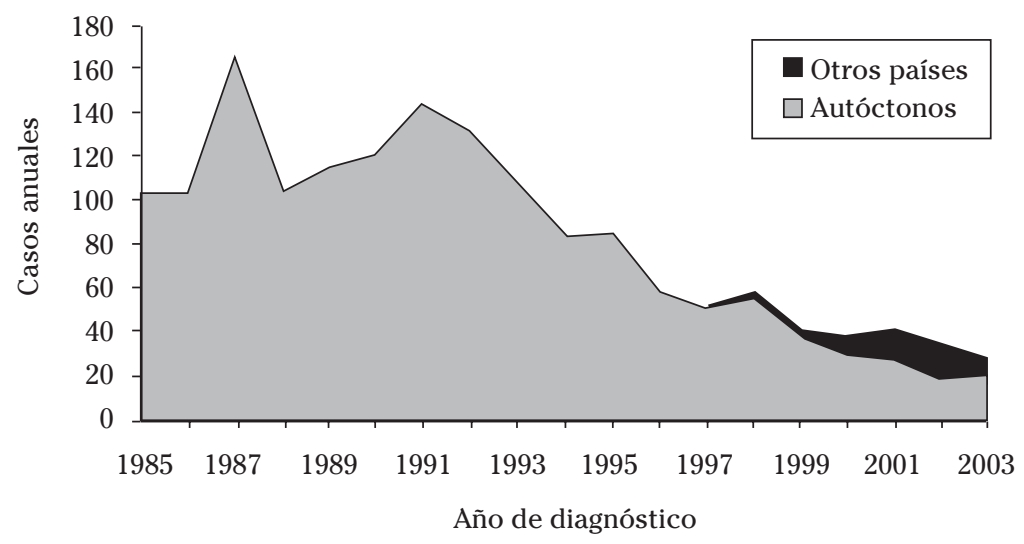

Figura 3. Tendencia de nuevos diagnósticos de infección por el VIH.

diagnósticos han aumentado ${ }^{14}$. Resultado de estas tendencias, Navarra ha pasado a presentar en los últimos años tasas de diagnósticos de VIH menores que muchos países de Europa Occidental (Fig. 4). También se ha descrito una evolución similar en otros lugares de España ${ }^{1,12}$.

\section{Características de los diagnósticos de VIH realizados en 2000-2003}

Con el fin de aproximarnos lo más posible a las características de las infecciones ocurridas recientemente, se han analizado los 141 diagnósticos de VIH realizados entre 2000 y 2003 . El $67 \%$ eran hombres y el $33 \%$ mujeres. El $61 \%$ de los hombres y el $91 \%$ de las mujeres tenían menos de 40 años (Fig. 5). Más de la mitad de las infecciones (58\%) eran atribuibles a la transmisión heterosexual, el 18\% se diagnosticaron en personas con antecedente de uso de drogas por vía parenteral y el $12 \%$ en hombres homosexuales. El 33\% de los diagnósticos de VIH se realizaron en personas originarias en otros países. Entre estos casos la proporción de mujeres fue mayor (44\%) que entre los casos autóctonos (28\%), y fue más frecuente la transmisión heterosexual (70\%) (Fig. 6).

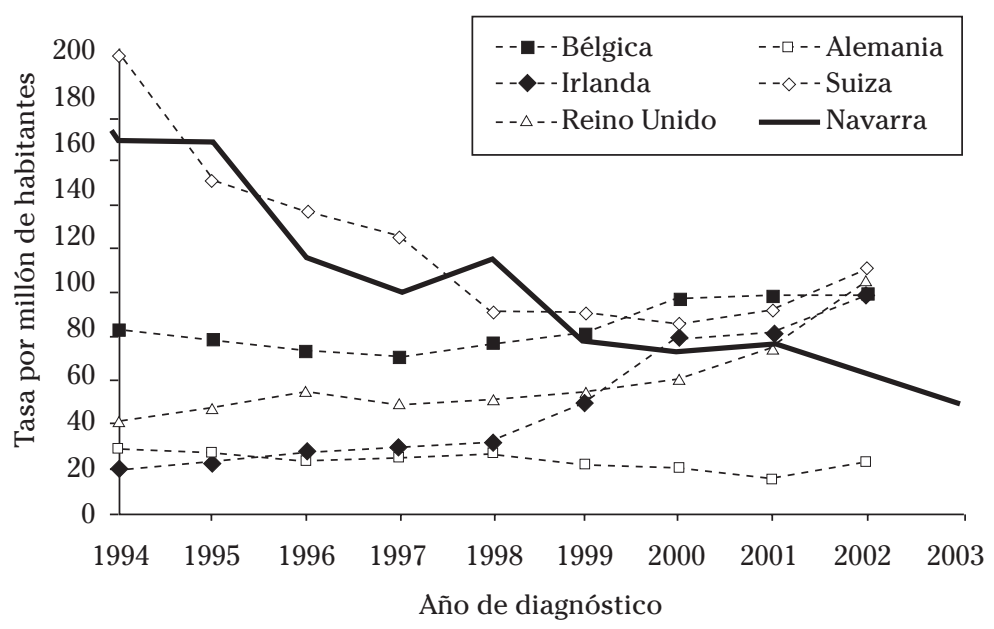

Figura 4. Tendencia de los nuevos diagnósticos de VIH en Navarra y en varios países europeos. 


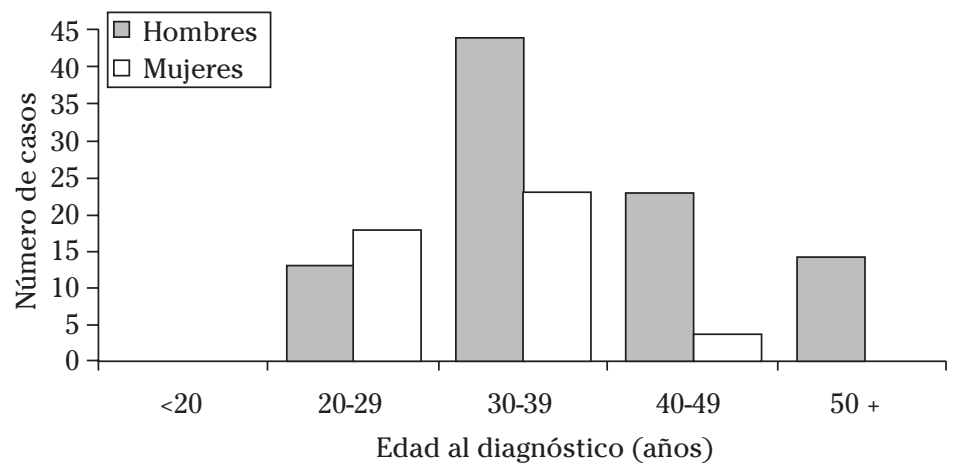

Figura 5. Diagnósticos de VIH según edad y sexo, 2000-2003.

En el 21\% se realizó un diagnóstico simultáneo de infección por el VIH y de sida. De estos casos simultáneos, el $69 \%$ eran atribuibles a transmisión heterosexual, el $17 \%$ se diagnosticaron en hombres homosexuales y sólo el $7 \%$ se diagnosticaron en personas con antecedente de uso de drogas por vía parenteral. En el 7\% restante no se pudo conocer el mecanismo probable de transmisión. El $31 \%$ de estos diagnósticos se realizaron en personas originarias de otros países.

\section{Personas diagnosticadas de infección por el VIH que permanecen vivas a finales de 2003}

La situación actual de la epidemia viene definida por la existencia de un elevado número de personas que viven con la infección por el VIH. Los avances en el tratamiento antirretroviral han mejorado notablemente la supervivencia, sin conseguir la curación, lo que convierte la infección por el VIH en un proceso tendente a la cronificación. Las nuevas terapias evitan buena parte de las enfermedades oportunistas que deterioraban la calidad de vida de las personas infectadas, pero al tener que mantenerse de forma indefinida surgen nuevos problemas como los efectos adversos, problemas de adherencia a los tratamientos y resistencias.

El número de personas vivas con infección por el VIH define la dimensión de la epidemia en cada momento. Una proporción de ellas permanece sin diagnosticarse y, por tanto, no se conocen sus características. A finales de 2003 había 902 personas vivas con diagnóstico de infección por el

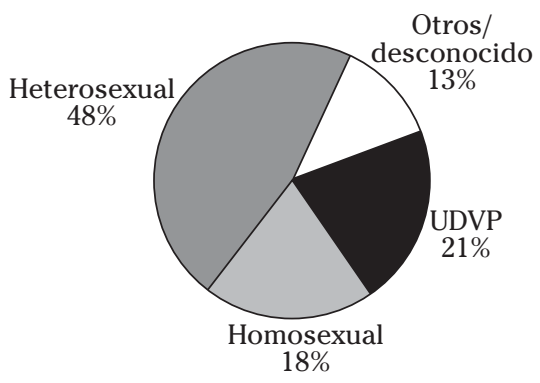

Hombres $(\mathrm{n}=95)$

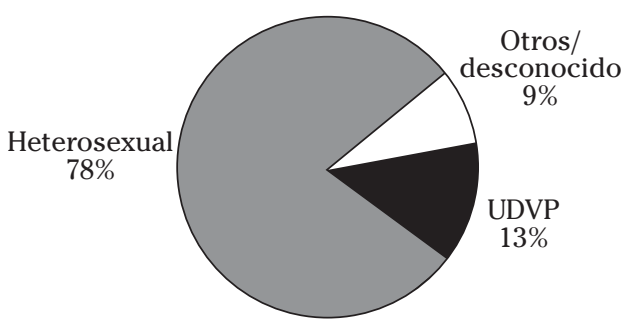

Mujeres $(\mathrm{n}=96)$

Figura 6. Distribución según sexo y categoría de transmisión de los diagnósticos de infección por el VIH, 2000-2003. 
$\mathrm{VIH}$, cifra que supone una prevalencia de 1,6 infecciones conocidas por cada 1.000 habitantes. De estas personas el 30\% han presentado en algún momento una enfermedad diagnóstica de sida. La tercera parte son mujeres y el $6 \%$ son personas originarias de otros países. En torno a dos terceras partes de estas personas adquirieron la infección por compartir material para la inyección de drogas, lo cual tiene importancia porque estas personas presentan también prevalencias altas de coinfección por el virus de la hepatitis C. Algo más de la cuarta parte habían adquirido el VIH por vía sexual.

\section{Reducción de la morbilidad y mortalidad por sida}

Muchas de las personas infectadas por el VIH en ausencia de tratamiento, tienden a evolucionar hacia un deterioro progresivo del sistema inmunitario. Los avances en el tratamiento antirretroviral pretenden evitar este deterioro del sistema inmune $y$, con ello, reducir la aparición de enfermedades oportunistas y la muerte. Un requisito previo para este objetivo es el diagnóstico de la infección por el VIH, ya que sólo las personas diagnosticadas pueden beneficiarse del tratamiento. Otros factores que pueden limitar la efectividad de los tratamientos antirretrovirales son la falta de adherencia, la aparición de resistencias y los efectos adversos. La incidencia de casos de sida y la mortalidad por esta causa son dos indicadores útiles para evaluar la efectividad real de las terapias antirretrovirales en la población de personas infectadas por el VIH.

En Navarra la incidencia de casos de sida aumentó hasta 1994, año en el que se produjeron 90 casos. Desde entonces, el principal descenso (41\%) se produjo entre 1996 y 1997, coincidiendo con la introducción de los tratamientos antirretrovirales de alta eficacia. En los años posteriores la incidencia de sida ha continuado una tendencia predominantemente descendente (Fig. 7, Tabla 1), aunque entre 2000 y 2003 todavía se diagnosticaron 94 nuevos casos de sida (tasa media anual de 4,2 por 100.000 habitantes). El 74,5\% de estos casos se produjeron en hombres y el $25,5 \%$ en mujeres. El 17\% eran personas originarias de otros países. Las enfermedades diagnósticas de sida más frecuentes en el período 2000-2003 han sido la neumonía por Pneumocystis carinii (21\%), la candidiasis esofágica (20\%), la tuberculosis (17\%) y la toxoplasmosis cerebral $(8,5 \%)$. Este patrón difiere del de la mayoría de las comunidades autónomas españolas, donde la tuberculosis es la enfermedad diagnóstica de sida más frecuente ${ }^{1}$.

Destaca el hecho de que el $40 \%$ de los casos de sida diagnosticados entre 2000 y 2003 desconocían estar infectados por el VIH hasta que debutaron con la enferme-

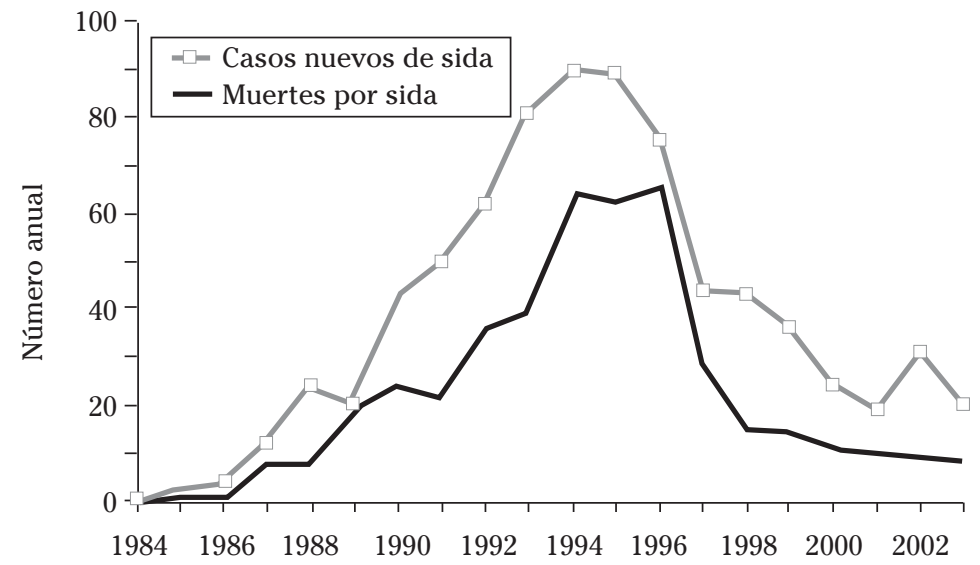

Figura 7. Evolución de la incidencia y mortalidad del sida en Navarra, 1984-2003. 
dad diagnóstica de sida. Este porcentaje superó el $60 \%$ entre los casos de sida que habían adquirido la infección por prácticas sexuales de riesgo (Fig. 8). Este retraso en el diagnóstico de la infección por el VIH impidió comenzar el tratamiento antirretroviral a tiempo para poder evitar la aparición de las enfermedades diagnósticas de sida ${ }^{16-17}$.

El impacto de la introducción de los tratamientos antirretrovirales de alta eficacia, fue todavía mayor en la mortalidad. Entre 1996 y 1998 la mortalidad por sida descendió en un $77 \%$, y desde entonces hasta 2003 ha disminuido en otro $47 \%$. Entre 2000 y 2003 todavía se produjeron 38 muertes entre los casos de sida, lo que supone una tasa media anual de 1,6 por cada 100.000 habitantes.

\section{Comportamientos relacionados con la infección por el VIH}

La monitorización de las conductas de riesgo para el VIH suele abordarse mediante la repetición de encuestas dirigidas a distintos grupos de población. A continuación se presentan resultados de dos encuestas, una dirigida a jóvenes y otra a usuarios de drogas inyectadas.

Según la encuesta realizada en 2003 en jóvenes navarros de 15 a 29 años, el nivel de información sobre el sida y las formas de prevenirlo fueron aceptables ${ }^{4}$. El 65\% habían mantenido al menos una vez rela- ciones sexuales coitales, y esta proporción llegaba al 88\% en el grupo de 25 a 29 años. La edad media de la primera experiencia coital se situaba en los 18,6 años.

El 86\% de los encuestados eran partidarios de utilizar el preservativo, y el 4,2\% se oponían a su uso. Entre los primeros, el $17 \%$ no lo usa siempre y un $26 \%$ no lo habían usado aún.

La razón más importante para el uso del preservativo es la prevención del embarazo (87\%), y el 58\% lo usan para la prevención de la infección por el VIH.

En comparación con encuestas de características similares realizadas en años anteriores, se observa una progresivo aumento en el porcentaje de jóvenes menores de 22 años que habían tenido alguna relación sexual coital (Fig. 9).

La encuesta realizada en 2002 a consumidores de drogas por vía intravenosa pone de manifiesto que las prácticas de riesgo siguen dentro de parámetros preocupantes. El 22\% había compartido jeringuillas recientemente y el $43 \%$ en el último año. Además, el $66 \%$ habían tenido relaciones sexuales de riesgo en el último año. Las diversas variables configuradoras del impacto de los programas de intercambio de jeringuillas no parecen influir en estos comportamientos, ni tampoco las que contemplan la valoración del dispositivo por parte de las personas usuarias del mismo. No obstante, la disponibilidad de material

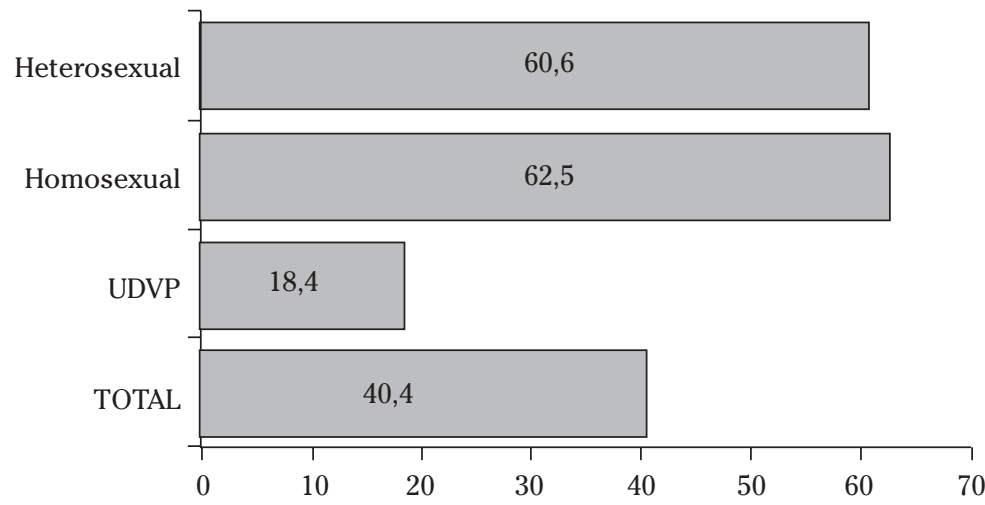

Figura 8. Casos de sida diagnosticados en 2000-2003 con diagnóstico simultáneo de su infección por el VIH. 


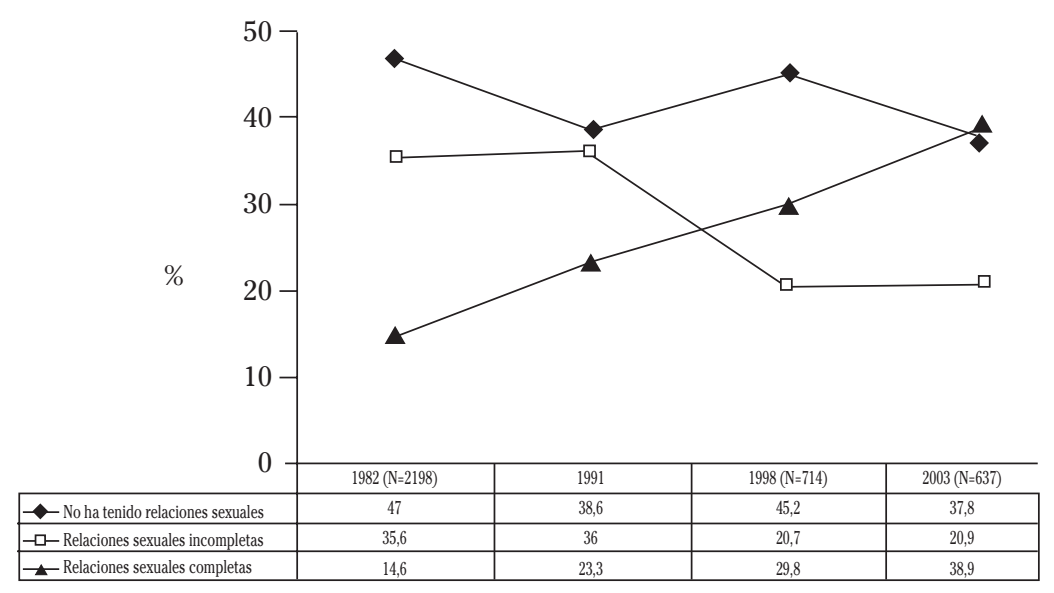

Figura 9. Actividad sexual en los jóvenes navarros de 15 a 21 años, 1982-2003.

de inyección en programas de intercambio de jeringuillas y en las oficinas de farmacia resulta un factor protector para reducir la práctica de compartir jeringuillas y otras prácticas de riesgo asociadas al uso inyectado de drogas. En esta misma encuesta se observa que la disponibilidad de jeringuillas continúa siendo un factor necesario para mantener bajos los niveles de prácticas de riesgo para la transmisión del VIH y los virus de la hepatitis $\mathrm{B}$ y $\mathrm{C}^{5}$. Sin embargo, estas estrategias no son suficientes para eliminar las prácticas de riesgo en el consumo inyectado ni en las relaciones sexuales en este colectivo. El conocimiento básico sobre la infección por el VIH y su forma de transmisión no implica tener las actitudes y habilidades precisas para aplicarlo en todas las situaciones con riesgo potencial.

\section{DISCUSIÓN}

La transmisión del VIH ha disminuido mucho respecto a épocas pasadas, pero todavía es elevada. El número de nuevas infecciones por transmisión sexual ha disminuido menos y actualmente supera a las de transmisión parenteral. Estos datos avalan el buen funcionamiento de los programas de prevención en Navarra, aunque todavía es preciso reforzarlos y adaptarlos a las nuevas situaciones.

Los tratamientos antirretrovirales han disminuido la mortalidad por sida, pero no han conseguido la curación definitiva de la infección. Esto lleva a que el número de personas que viven con la infección por el VIH se mantenga en cifras elevadas, a pesar de los avances en la prevención. Al menos 9 de cada 10 pacientes con VIH están en seguimiento en servicios médicos especializados en el manejo de esta infección.

Las personas infectadas por el VIH que no han sido diagnosticadas suponen una dificultad para conseguir un mayor impacto de los tratamientos antirretrovirales y de las actividades de prevención. Convendría, por ello, aumentar la frecuencia de realización de la prueba de VIH mediante su promoción de manera oportunista $\mathrm{y}$ mejorar la accesibilidad a la misma.

El creciente porcentaje de inmigrantes entre las personas con diagnóstico de $\mathrm{VIH}$ hace necesario estimular el acercamiento de estas personas a las actividades de información y prevención, y mejorar su accesibilidad a los servicios sanitarios, reduciendo las barreras lingüísticas, culturales y administrativas.

Aunque en menor medida, todavía se detectan infecciones por VIH entre usuarios de drogas por vía parenteral. Estudios realizados en nuestro medio han demostrado la eficacia y eficiencia de los programas de dispensación e intercambio de jeringuillas para la prevención del VIH y 
otras infecciones de transmisión por parenteral $^{18}$. Su eficacia es mayor cuando se alcanza mayor cobertura, por lo cual, uno de los objetivos debe ser aumentar la cobertura y la captación para el programa de personas que usan drogas por vía parenteral.

En relación con el VIH y sida todavía existen muchos temas que deben ser objeto de investigación: la búsqueda de soluciones a los problemas de salud de las personas con larga evolución de la infección por VIH, el estudio de factores que pueden mejorar la adherencia al tratamiento, los condicionantes de la calidad de vida en las personas con infección por el $\mathrm{VIH}$, la evaluación de la efectividad de los programas de prevención en jóvenes, las nuevas situaciones surgidas en torno a las poblaciones de emigrantes y la evaluación económica de diferentes estrategias de intervención.

\section{BIBLIOGRAFIA}

1. Secretaría del Plan Nacional sobre el sida. VIH y sida en España, Situación epidemiológica 2001. Madrid: Ministerio de Sanidad y Consumo 2002.

2. Moreno C, Irisarri F, Urtiaga M, Sola J, FerNÁNDEZ C, MARTíneZ V et al. Vigilancia epidemiológica de las infecciones por el VIH /sida en Navarra entre 1985 y 2002. An Sist Sanit Navar 2003; 26: 269-275.

3. Secretaría del Plan Nacional sobre el Sida. Infección por el VIH y sida en España. Plan de multisectorial, 2001-2005. Madrid: Ministerio de Sanidad y Consumo 2001.

4. Fundación Bartolomé de Carranza. Juventud Navarra 2003. V encuesta sobre la juventud Navarra. Pamplona: Fundación Bartolomé de Carranza 2004.

5. Fundación Bartolomé de Carranza. Evaluación pluridisciplinar de los dispositivos de suministro e intercambio de jeringuillas en Navarra. Síntesis y conclusiones. Pamplona: Fundación Bartolomé de Carranza 2002.

6. HAMERS FF, for the group of experts and national coordinators of HIV/AIDS surveillance from the countries of WHO European Regions. Recommendations for HIV surveillance in Europe. Eurosurveillance 1998; 3: 51.

7. Infuso A, Hamers FF, Downs AM, Alix J. HIV reporting in western Europe: national sys- tems and first European data. Eurosurveillance 2000; 5: 13-17.

8. Moreno C, Huertas I, Lezaun me, Gonzalez A, Sola J, Castilla J. Evolución del número de nuevos diagnósticos de infección por el VIH en Asturias, Navarra y La Rioja. Med Clin (Barc) 2000; 114: 653-655.

9. Centers for Disease Control and Prevention. Guidelines for national human immunodeficiency virus case surveillance, including monitoring for human immunodeficiency virus infection and acquired immunodeficiency syndrome. MMWR 1999; 48(RR13): 128.

10. Gostin OL, WARD JW, BAKER AC. National VIH case reporting for the United States. N Engl J Med 1997; 337: 1162-1166.

11. AnCELLE-PARK RM. Expanded European AIDS case definition. Lancet 1993; 341:441.

12. GonzÁlez-CElador R, SACRISTÁN-Salgado A, VAlero LF, SÁEnz-GonzÁlez MC. Epidemiología de la infección por el virus de la inmunodeficiencia humana (VIH) en la provincia de Salamanca (1985-2002). Enf Infecc Microbiol Clin 2004; 22: 272-278.

13. Zulaika D, Aguirrebengoa K, Andía A, ArrizaBalaga J, Bustillo JM, CÁMARA MM et al. Características epidemiológicas de las nuevas infecciones causadas por el VIH comparadas con los casos de sida. La epidemia de VIH/sida en el País Vasco. Gac Sanit 2004; 18: 145-149.

14. European Centre for the Epidemiological Monitoring of AIDS. HIV/AIDS Surveillance in Europe.Mid-year report 2003. Saint-Maurice:Institut de Veille Sanitaire 2003. No. 69.

15. Secretaría del Plan Nacional sobre el Sida. Prevención del VIH/sida en inmigrantes y minorías étnicas. Madrid: Ministerio de Sanidad y Consumo 2001

16. Castilla J, Sobrino P, de la Fuente L, Noguer I, GuERrA L, PARRAS F. Late diagnosis of HIV infection in the era of highly active antiretroviral therapy: consequences on AIDS incidence. AIDS 2002; 16: 1945-1951.

17. Castilla J, Noguer I, Belza MJ, del Amo J, SÁNCHEz F, GUERRA L. ¿Estamos diagnosticando a tiempo a las personas infectadas por el VIH? Aten Prim 2002; 29: 20-25.

18. Cabases JM, SÁnchez E. Cost and effectiveness of a syringe distribution an needle exchange program for HIV prevention in a regional setting. Eur J Health Econom 2003; 4: $203-208$ 
